\title{
ĐÁNH GIÁ CHUẨN ĐẦU RA THEO KIỂM ĐỊNH ABET ÁP DỤNG CHO CHƯƠNG TRÌNH KỸ THUẬT DẦU KHÍ CỦA TRƯỜNG ĐẠI HỌC DẦU KHÍ VIẸT NAM
}

\author{
Nguyễn Văn Hùng ${ }^{(*)}$, Trương Thanh Tuấn ${ }^{(*)}$, Bùi Tử An ${ }^{(* *)}$ \\ (*) Tiến sĩ. Truờng Đại học Dầu khí Việt Nam. Email: hungnv@pvu.edu.vn. \\ (**)Thạc sĩ. Truờng Đại học Dầu khí Việt Nam.Email: anbt@pvu.edu.vn.
}

DOI: $10.37550 /$ tdmu.CFR/2021.01.128

\section{Tóm tắt}

Bài báo này mục đích giới thiệu các thông tin chung về bộ tiêu chuẩn ABET và đặc biệt tập trung vào đánh giá chuẩn đầu ra. Trên co sở phân tích các chuơng trình kỹ thuật trên thế giới và Việt Nam, nhóm tác giả thục hiện lựa chọn quy trình phù hợp để áp dụng cho chuơng trình kỹ thuật dầu khí của Truòng Đại học Dầu khi Việt Nam. Kết quả của nghiên cưu cho thấy, quy trình đánh giá ABET giúp tạo ra một chu trình cải tiến hoàn chỉnh và người hoc có thể được đón nhận một sản phẩm tốt, chuẩn bị chu đáo khi bước vào thị truờng lao động tại Việt Nam và trên thế giới. Phuơng pháp đánh giá trực tiếp và gián tiếp trong 1 năm cho kết quả khá tuoong đồng và đạt kì vọng đặt ra. Bên cạnh đó, quá trình đánh giá cũng đưa ra một số giải pháp cải tiến cho một số học phần chưa đạt chuẩn đầu ra của học phần cho giai đoạn tiếp theo.

Từ khóa: Kỹ thuật dầu khí, ABET, Chuẩn đầu ra, CDIO, AUN-QN.

\section{1. Đặt vấn đề}

Hội đồng Kiểm định Kỹ thuật và Công nghệ ABET (Accrediation Board for Engineering and Technology) là một tổ chức kiểm định chất lượng các chương trình đào tạo có uy tín trong cộng đồng quốc tế. Đây là một tổ chức phi chính phủ, mang tính trung gian và được Bộ Giáo dục Hoa Kỳ, Hội đồng kiểm định giáo dục đại học Hoa Kỳ công nhận từ năm 1997 [1]. Đến năm 2020, đã có 4.307 chương trình của 864 trường đại học và cao đẳng ở 41 quốc gia được kiểm định và công nhận. Hiện nay có 04 Hội đồng (Uỷ Ban) kiểm định, bao gồm: (1) Uỷ ban Kiểm định các chương trình kỹ thuật (EAC - Engineering Accrediatation Commission), (2) Uỷ ban Kiểm định các chương trình Công nghệ Kỹ thuật (ETAC - Engineering Technology Accreditation Commission), (3) Uỷ ban Kiểm định các chương trình máy tính (CAC Computing Accreditation Commission), (4) Uỷ ban Kiểm định các chương trình Khoa học tự nhiên ứng dụng (ANSAC - Applied and Natural Science Accreditation Accommission) [2]. Quy trình kiểm định của $\mathrm{ABET}$ là rất nghiêm ngặt cho 09 tiêu chuẩn chung và riêng (tiêu chuẩn Ngành), cụ thể: (1) Sinh viên, (2) Mục tiêu chương trình đào tạo, (3) Chuẩn đầu ra sinh viên, (4) Cải tiến liên tục, (5) Chương trình đào tạo, (6) Giảng viên, (7) Cơ sở vật chất, (8) Sự hỗ trợ từ Nhà trường, (9) Tiêu chí của chương trình. 
Một chương trình đạt kiểm định $\mathrm{ABET}$ phải trải qua một quá trình lâu dài và kế hoạch hành động cụ thể trong từng giai đoạn. Thứ nhất, Ban giám hiệu của cơ sở giáo dục cần quan tâm đặc biệt, thực hiện đồng bộ các điều kiện cho chương trình đào tạo, quan hệ giữa các bên liên quan (Sinh viên, Giảng viên, Doanh nghiệp, Hội đồng khoa học khoa/trường). Thứ hai, cơ sở giáo dục cần lên chiến lược và kế hoạch kiểm định cụ thể, có kinh phí đầu tư cơ sở vật chất, xây dựng hệ thống đánh giá và cải tiến chất lượng một cách liên tục. Cuối cùng, chương trình đào tạo cần được xây dựng bài bản, ví dụ theo phương pháp tiếp cận CDIO [3] và đào tạo, đánh giá chính xác người học như cam kết trong mục tiêu chương trình đào tạo, chuẩn đầu ra sinh viên. Nhưng ngược lại thì lợi ích mang lại của chương trình đạt $\mathrm{ABET}$ cho người học là vô cùng to lớn, mang lại cơ hội và sự thừa nhận trên toàn thế giới [4].

Vấn đề tuyển sinh của các cơ sở giáo dục hiện nay đều gặp nhiều khó khăn. Nguyên nhân của việc này một phần là hiện có quá nhiều lựa chọn nơi học trong nước và nước ngoài. Vì vậy, để thu hút được sự quan tâm phụ huynh và học sinh, Nhà trường/Chương trình đào tạo phải có điểm nổi bật có với các chương trình của trường khác, cũng như tạo được những cơ hội cho người học sau khi tốt nghiệp [5]. Hay nói cách khác, chương trình đào tạo của một cơ sở giáo dục muốn có nhiều người theo học thì cần khẳng định được giá trị tại khu vực hoặc trên toàn cầu. Một trong những lựa chọn ưu tiên trong thời gian gần đây là kiểm định $\mathrm{ABET}$, bởi lẽ điểm nổi bật là được công nhận trên phạm vi toàn cầu so với kiểm định AUN (trong khu vực Châu Á) hay của Bộ Giáo dục và Đạo tạo (mang tính quốc gia).

Tuy nhiên, một chương trình đào tạo muốn theo đuổi và đạt được chuẩn kiểm định ABET là điều không dễ, hơn nữa là trong điều kiện tài liệu hướng dẫn phù hợp với điều kiện của Việt Nam là không nhiều. Bài báo này viết với mục đích chia sẻ những thông tin chuẩn bị của chương trình kỹ thuật dầu khí của Trường Đại học Dầu khí Việt Nam trong quá trình chuẩn bị kiểm định $\mathrm{ABET}$ dưới sự tư vấn của các chuyên gia hàng đầu, các kiến thức thu được từ các khoá đào tạo về $\mathrm{ABET}$. Do tổng cộng có 9 tiêu chuẩn trong bộ tiêu chí kiểm định $\mathrm{ABET}$, nên bài báo lựa chọn tiêu chuẩn đánh giá chuẩn đầu ra (Student Outcomes) vì đây được coi là một trong những tiêu chuẩn quan trọng và cốt lõi của $\mathrm{ABET}$.

\section{Phương pháp tiếp cận}

Để lựa chọn được quy trình đánh giá chuẩn đầu ra cần nghiên cứu kĩ nội hàm và cách thực hiện của các chương trình tương đồng đã đạt kiểm định $\mathrm{ABET}$. Do vậy, quá trình phân tích để làm nổi bật kết quả đạt được của người học so với kỳ vọng của ban thiết kế, thực hiện chương trình đào tạo là rất quan trọng để từ đó đưa ra quy trình phù hợp để cải tiến chương trình với sự tham gia của các bên liên quan. Tuy nhiên, sẽ có những khó khăn trong quá trình thiết kế và xây dựng quy trình đánh giá cần được cân nhắc khi cải tiến chất lượng chương trình đào tạo, bao gồm:

\subsection{Tính toàn diện và đơn giản vấn đề}

Điều quan trọng trong khi nghiên cứu quy trình đánh giá chuẩn đầu ra là cần xem xét đầy đủ và cân đối giữa các điểm: chương trình, giảng viên, người học, công cụ đánh giá, đề xuất khả thi, hỗ trợ của cơ sở. Phương pháp đơn giản vấn đề là cần đưa ra quy trình khả thi 
nhất, rõ ràng nhất dưới sự hỗ trợ của nguồn lực hiện tại của Nhà trường và đánh giá được tác động của phương pháp đó tới kết quả thu được.

\subsection{Phương pháp đánh giá}

Đây là nội dung cốt lõi của quy trình đánh giá vì các công việc tiếp theo trong quy trình đều dựa trên cơ sở kết quả của phương pháp đề ra. Trong khi tiếp cận theo phương pháp từ trên xuống (Top Down) được thực hiện theo đúng trình tự của quy trình thiết kế chương trình, đó là từ bộ phận quản lý tới người thực hiện, còn phương pháp từ dưới lên (Bottom Up) lại thực hiện ngược lại với điểm xuất phát là từ các bên liên quan. Nhưng dù là tiếp cận theo phương pháp nào thì vẫn cần xem xét đầy đủ các mặt: Mục tiêu chương trình đào tạo (PEO - Program Educational Objectives), Chuẩn đầu ra (SOs - Student Outcomes), Chỉ báo (PIs - Performance Indicators) và thiết kế phù hợp cách đánh giá trực tiếp, gián tiếp, cũng như bảng đánh giá các mặt (Rubrics), tuần suất đánh giá.

\subsection{Công tâm/Không tập trung}

Phương pháp đánh giá phải công tâm và không được quá tập trung vào một đối tượng nào để đưa ra được kết quả phù hợp, chính xác. Phải đảm bảo có sự tham gia của đầu đủ các bên liên quan, gồm: người học, giảng viên, doanh nghiệp, hội đồng khoa học, để đảm bảo đáp ứng các mặt kinh tế - xã hội, địa lý và thị trường lao động.

\subsection{Tham gia của giảng viên}

Giảng viên là người triển khai thực tế chương trình giảng dạy tới sinh viên, vì vậy đây phải là đội ngũ đầu tiên tham gia vào quá trình đánh giá chuẩn đầu ra. Trong quá trình giảng dạy, giảng viên sẽ theo dõi và ghi nhận bằng cách cho điểm qua các hình thức. Đồng thời hình thức đánh giá theo các tiêu chí (Rubrics) với sự tham gia góp ý của các bên cũng là công cụ tốt để các giảng viên sử dụng khi cho điểm.

\subsection{Quản lý hỗ trọ}

Đội ngũ hỗ trợ là rất quan trọng trong quá trình thiết kế và thực hiện hiện quả các quy trình đánh giá. Có nhiều khía cạnh khi đề cập tới đội ngũ này, nhưng cốt lõi là nguồn lực phù hợp và mức độ tham gia vào quy trình. Bên cạnh đó, cần vạch rõ sự tham gia ở mức độ nào hay nhiệm vụ của họ vào quá trình thiết kế, thực thi, rà soát, cải tiến PEOs, SOs, PIs, khung rubrics. Ngoài ra, đội ngũ quản lý cũng đóng vai trò quan trọng trong quá trình thu thập và xử lý phản hồi của các bên liên quan tham gia.

\subsection{Dễ dàng kiểm tra}

Phương pháp đánh giá cần cho ra được kết quả định lượng của sinh viên đạt được, đồng thời đối sánh với mục tiêu đề ra. Tuy nhiên, đây là công việc khó vì dữ liệu đánh giá không chỉ lấy trực tiếp mà còn lấy từ phương pháp gián tiếp trong những giai đoạn khác nhau, ở các mức hay thước đo khác nhau.

\subsection{Cải tiến liên tục}

Đây là kết quả quan trọng nhất trong quy trình đánh giá vì cần đưa ra được dữ liệu sau xử lý, phản hồi và đề xuất cải tiến liên tục sau một vòng thực hiện. Điều này có nghĩa là 
quy trình đánh giá phải được thiết kế theo cách có kết quả tạo ra là sự kế thừa cho giai đoạn tiếp theo để thực hiện.

\section{Kết quả và đánh giá}

\subsection{Chưong trình đào tạo ngành Kỹ thuật dầu khí}

Bài báo này sẽ phân tích kết quả đánh giá chuẩn đầu ra theo ABET (phiên bản mới, có 07 chuẩn đầu ra, SO1-SO7) của Uỷ ban kỹ thuật EAC cho chương trình kỹ thuật dầu khí, chuyên ngành "Khoan - Khai thác dầu khí" trường Đại học Dầu khí Việt Nam. Chương trình đào tạo này được xây dựng từ năm 2010, đào tạo hệ kỹ sư với thời gian 5 năm. Sau đó, chương trình được rút gọn xuống thời gian đào tạo 4 năm kể từ năm 2016 và hiện tại đã có 01 khoá tốt nghiệp ra trường. Uu điểm của chương trình là được xây dựng bởi tư vấn tổng thể của hiệp hội tư vấn Hoa Kỳ (TIEC) trên cơ sở yêu cầu của ABET (theo hệ cũ, gồm 11 tiêu chuẩn đầu ra $\mathrm{a}-\mathrm{k}$ ). Số tín chỉ tương ứng giảm từ 160 còn 154 và tương ứng số học phần là 70,64 . Tất cả các thông tin về chương trình được công khai trên trang web tiếng anh của Nhà trường, phần chương trình kỹ thuật dầu khí theo đúng yêu cầu của ABET về sự công khai, cam kết với cộng đồng.

\subsection{Mục tiêu chuơng trình đào tạo kỹ thuật dầu khí}

Mục tiêu của chương trình kỹ thuật dầu khí là đào tạo cho sinh viên sau 5 năm tốt nghiệp có khả năng:

- Áp dụng kiến thức chuyên sâu, thiết kế, thi công, sản xuất, nghiên cứu, sáng tạo và quản lý sản xuất trong ngành đặc thù

- Tiếp tục phát triển chuyên môn và trở thành nhà quản lý trong các công ty thành viên của Tập đoàn dầu khí Việt Nam, các công ty đa quốc gia và trở thành các nhà khoa học nghiên cứu tại các viện và trường đại học

- Làm việc độc lập hoặc nhóm, giao tiếp hiệu quả, tư cách đạo đức, tư duy phản biện, sức khoẻ, cũng như khả năng học tập suốt đời trong môi trường phát triển hiện đại của nền công nghiệp dầu khí, đối mặt các thách thức trong môi trường lao động năng động

\subsection{Chuẩn đầu ra của chương trình kỹ thuật dầu khí}

Các chuẩn đầu ra của chương trình được công bố trên trang web tiếng anh của bộ môn, được phổ biến rộng rãi tới sinh viên trong quá trình học. Cụ thể, sinh viên tại thời điểm tốt nghiệp có khả năng:

- Nhận diện, xây dựng công thức và giải các bài toán kỹ thuật phức tạp bằng cách sử dụng các kiến thức toán, khoa học và kỹ thuật (SO1)

- Áp dụng thiết kế kỹ thuật để đưa ra lời giải cho bài toán gặp phải và có tính tới các yếu tố sức khoẻ cộng đồng, an toàn, phúc lợi, cũng như tính chất toàn cầu, văn hoá, xã hội, môi trường ( $\mathrm{SO} 2)$

- Giao tiếp hiệu quả với nhiều đối tượng khác nhau (SO3) 
- Ý thức trách nhiệm đạo đức, trách nhiệm nghề nghiệp trong các tình huống kĩ thuật và đưa ra những đánh giá sáng suốt, xem xét tác động của các giải pháp kĩ thuật trong bối cảnh toàn cầu, kinh tế, môi trường và xã hội (SO4)

- Làm việc hiệu quả trong một nhóm mà thành viên cùng nhau tham gia lãnh đạo nhóm, tạo ra môi trường hợp tác và hoà thuận, thiết lập mục tiêu, lập kế hoạch và đáp ứng các mục tiêu (SO5)

- Phát triển và thực hiện các thí nghiệm phù hợp, phân tích và minh giải dữ liệu và sử dụng phán đoán kĩ thuật để đưa ra kết luận (SO6)

- Tiếp thu và áp dụng kiến thức mới khi cần thiết, sử dụng các chiến lược học tập phù hợp (SO7).

\subsection{Quy trình cải tiến liên tục}

Mục đích chính của việc xây dựng quy trình cải tiến liên tục là cung cấp phương pháp tiếp cận hệ thống để định lượng mức độ đạt được của 07 chuẩn đầu ra.

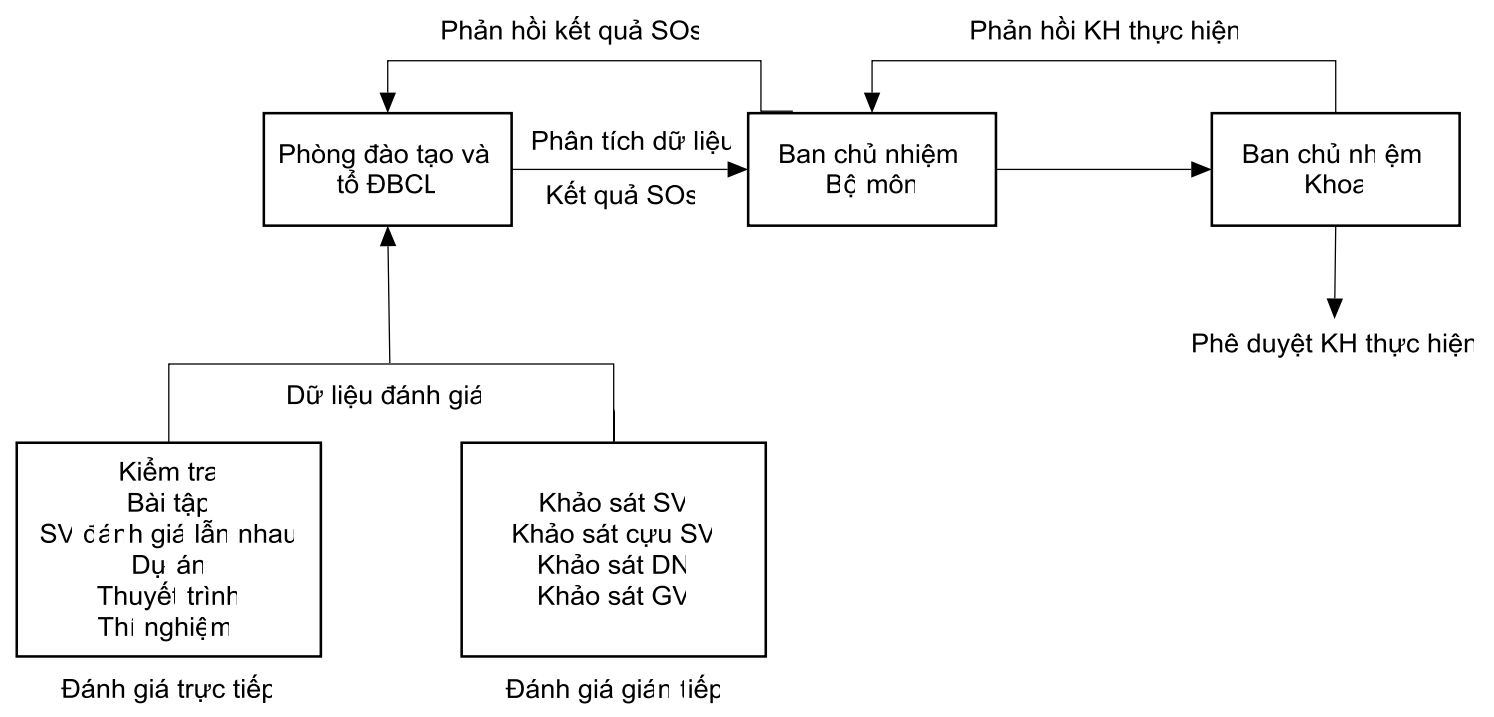

Hình 1. Quy trình đánh giá chuẩn đầu ra

Cải tiến liên tục là một trong những nội dung ưu tiên của Bộ môn khoan - khai thác dầu khí trong quá trình vận hành chương trình. Mục tiêu là khắc phục những điểm bất cập được tìm ra khi rà soát kết quả thông qua đánh giá các chuẩn đầu ra. Các điều chỉnh thông qua đề cương chi tiết để làm rõ nội dung giảng dạy, từ đó hướng tới người học đạt được mục tiêu chương trình đào tạo.

Thông qua quy trình đề xuất như hình 1 , thực tế triển khai cho các học kỳ và dữ liệu thu thập từ nguồn trực tiếp và gián tiếp. Với nguồn trực tiếp, giảng viên hay nhóm giảng viên trực tiếp giảng dạy học phần sẽ trao đổi thống nhất cách thức đánh giá sinh viên thông qua: kiểm tra giữa kỳ, bài tập, dự án, thuyết trình, bài thí nghiệm, sinh viên đánh giá lẫn nhau, với nguyên tắc đảm bảo đánh giá đầy đủ trong 3 thời điểm của học kỳ (điểm quá trình, điểm giữa kỳ, điểm cuối kỳ). Để đảm bảo khách quan thì toàn bộ điểm sẽ được lưu tại Phòng Đào tạo, đồng thời mỗi giảng viên đều có mục lưu trữ tài liệu của học phần do mình đảm nhận. Trong khi đó, đối với dữ liệu gián tiếp được thu thập từ khảo sát với nội dung bám sát chuẩn đầu ra của học 
phần/chương trình đào tạo thông qua các câu hỏi trong phiếu từ các bên: Sinh viên, Cựu sinh viên, Doanh nghiệp, Giảng viên. Công việc thực hiện do Phòng đào tạo đảm nhận, với nội dung câu hỏi thống nhất với giảng viên. Sau khi có dữ liệu từ Phòng đào tạo cung cấp, giảng viên sẽ xử lý và so sánh với kỳ vọng đặt ra trước đó của mỗi chuẩn đầu ra/Chỉ số (SOs/PIs). Báo cáo kết quả của giảng viên cho mỗi học phần sẽ trình Ban chủ nhiệm Bộ môn để trao đổi với Phòng đào tạo/Tổ ĐBCL. Sau khi thống nhất, kết quả cuối cùng kèm đề xuất hiệu chỉnh sẽ trình lên ban chủ nhiệm khoa để phản hồi triển khai và hiệu chỉnh nếu có.

\subsection{Kế hoạch cải tiến}

\subsubsection{Các học phần lấy minh chứng}

Trước mỗi học kỳ Ban chủ nhiệm khoa/Bộ môn sẽ họp để thống nhất học phần sẽ được lựa chọn để đánh giá $\mathrm{SO} / \mathrm{PI}$. Nguyên tắc của việc triển khai dựa trên: mỗi giảng viên không đánh giá quá 2-3 SO, mỗi $\mathrm{SO}$ sẽ lựa chọn tối đa 3 học phần và dải đều mức độ từ giới thiệu - tăng cường - nâng cao cho các năm 2 tới 4 . Kế hoạch cụ thể như bảng 1 .

Bảng 1: Các học phần lựa chọn đánh giá chuẩn đầu ra

\begin{tabular}{|c|c|c|c|c|c|c|c|c|}
\hline \multirow{2}{*}{ STT } & \multirow{2}{*}{ Học phần } & \multicolumn{7}{|c|}{ Chuẩn đầu ra } \\
\hline & & SO1 & $\mathrm{SO2}$ & SO3 & SO4 & SO5 & SO6 & SO7 \\
\hline \multicolumn{9}{|c|}{ Học kỳ I - Năm 2} \\
\hline 1 & PET12443 - Hệ thống khoan (+TH) & $\mathrm{X}$ & & $\mathrm{X}$ & & & & \\
\hline \multicolumn{9}{|c|}{ Học kỳ II - Năm 2} \\
\hline 2 & PET12404 - Vật lý vỉa (+TH) & & & & & $\mathrm{X}$ & $\mathrm{X}$ & \\
\hline 3 & $\begin{array}{l}\text { PET12306 - Quá trình vận chuyển } \\
\text { trong khai thác dầu khí }\end{array}$ & & $\mathrm{X}$ & & & & & \\
\hline \multicolumn{9}{|c|}{ Học kỳ I - Năm 3} \\
\hline 4 & PET13309 - Chất lưu vỉa & & & & & & $\mathrm{X}$ & \\
\hline 5 & $\begin{array}{l}\text { PET13411 - Hệ thống khai thác dầu } \\
\text { khí }(+\mathrm{TH})\end{array}$ & & & & & & & $\mathrm{X}$ \\
\hline 6 & PET13312 - Kỹ thuật khoan & & $\mathrm{X}$ & & & & & \\
\hline \multicolumn{9}{|c|}{ Học kỳ II - Năm 3} \\
\hline 7 & $\begin{array}{l}\text { PSE13201 - An toàn và môi trường } \\
\text { trong công nghiệp dầu khí }\end{array}$ & & & & $\mathrm{X}$ & & & \\
\hline 8 & PET13116 - Đồ án chuyên ngành 1 & & & $\mathrm{X}$ & & $\mathrm{X}$ & & \\
\hline \multicolumn{9}{|c|}{ Học kỳ I - Năm 4} \\
\hline 9 & PET14320 - Kỹ thuật khai thác & $\mathrm{X}$ & & & & & & \\
\hline 10 & PET14133 - Đồ án chyên ngành 2 & & & & $\mathrm{X}$ & $\mathrm{X}$ & $\mathrm{X}$ & $\mathrm{X}$ \\
\hline \multicolumn{9}{|c|}{ Học kỳ II - Năm 4} \\
\hline 11 & PET14825 - Đồ án tốt nghiệp & $\mathrm{X}$ & $\mathrm{X}$ & $\mathrm{X}$ & $\mathrm{X}$ & & & $\mathrm{X}$ \\
\hline
\end{tabular}

\subsubsection{Phưong pháp đánh giá trục tiếp}

Đối với đánh giá trực tiếp cho từng học phần như bảng 2.

1. Kiểm tra: Đánh giá trên câu hỏi giữa kỳ và cuối kỳ

2. Bài tập: Trong quá trình học của sinh viên

3. Sinh viên đánh giá lẫn nhau: Dựa trên các tiêu chí Rubrics, áp dụng cho làm việc nhóm 
Trường Đại học Thủ Dầu Một - Trường Đại học Duy Tân

4. Đánh giá năng lực quản lý: Bảng đánh giá tiêu chí (Rubrics)

5. Báo cáo bài tập lớn: Nhóm sinh viên thực hiện và có tiêu chí cụ thể đánh giá kĩ năng viết

6. Đánh giá bài thuyết trình: Tiêu chí đánh giá cho các kĩ năng giao tiếp trong thuyết trình

7. Thí nghiệm: Các tiêu chí đánh giá cho việc phân tích số liệu và minh giải dữ liệu.

Bảng 2: Phuơng pháp đánh giá chuẩn đầu ra cho chuơng trình kỹ thuật dầu khí

\begin{tabular}{|c|c|c|c|c|c|c|c|c|}
\hline \multirow{2}{*}{ STT } & \multirow{2}{*}{ Học phần } & \multicolumn{7}{|c|}{ Chuẩn đầu ra } \\
\hline & & SO1 & $\mathrm{SO2}$ & SO3 & SO4 & SO5 & SO6 & SO7 \\
\hline \multicolumn{9}{|c|}{ Học kỳ I - Năm 2} \\
\hline 1 & PET12443 - Hệ thống khoan (+TH) & 1,2 & & 5,6 & & & & \\
\hline \multicolumn{9}{|c|}{ Học kỳ II - Năm 2} \\
\hline 2 & PET12404 - Vật lý vỉa (+TH) & & & & & 3,4 & 7 & \\
\hline 3 & $\begin{array}{l}\text { PET12306 - Quá trình vận chuyển } \\
\text { trong khai thác dầu khí }\end{array}$ & & 6 & & & & 2 & \\
\hline \multicolumn{9}{|c|}{ Học kỳ I - Năm 3} \\
\hline 4 & PET13309 - Chất lưu vỉa & & & & & & 6 & \\
\hline 5 & $\begin{array}{l}\text { PET13411 - Hệ thống khai thác dầu } \\
\text { khí }(+\mathrm{TH})\end{array}$ & & & & & & & 2 \\
\hline 6 & PET13312 - Kỹ thuật khoan & & 1 & & & & & \\
\hline \multicolumn{9}{|c|}{ Học kỳ II - Năm 3} \\
\hline 7 & $\begin{array}{l}\text { PSE13201 - An toàn và môi trường } \\
\text { trong công nghiệp dầu khí }\end{array}$ & 1,2 & & & 5 & & & \\
\hline 8 & PET13116 - Đồ án chuyên ngành 1 & & & 5,6 & & 3,4 & & \\
\hline \multicolumn{9}{|c|}{ Học kỳ I - Năm 4} \\
\hline 9 & PET14320 - Kỹ thuật khai thác & 1 & & & & & & \\
\hline 10 & PET14133 - Đồ án chyên ngành 2 & & & & 5 & 3,4 & 2 & 2 \\
\hline \multicolumn{9}{|c|}{ Học kỳ II - Năm 4} \\
\hline 11 & PET14825 - Đồ án tốt nghiệp & 2 & 2 & 5,6 & 5 & & & 2 \\
\hline
\end{tabular}

\subsubsection{Phương pháp đánh giá gián tiếp}

Để thực hiện phương pháp này, phòng đào tạo đã phối hợp cùng thầy cô giảng dạy thống nhất nội dung khảo sát cho các đối tượng liên quan phù hợp. Các bản đánh giá được thực hiện bởi phòng đào tạo và chuyển dữ liệu cho giảng viên xử lý, đối sánh với kỳ vọng đặt ra như trong bảng 3 .

Bảng 3: Công cu đánh giá gián tiếp, thời gian đánh giá và kì vọng đạt được của sinh viên

\begin{tabular}{|c|c|c|c|}
\hline Công cụ & Tần suất đánh giá & Phương pháp & Kì vọng \\
\hline Khảo sát sinh viên & Theo học kỳ & Gián tiếp & $70 \%$ \\
\hline $\begin{array}{c}\text { Khảo sát sinh viên tốt } \\
\text { nghiệp }\end{array}$ & Theo năm học & Gián tiếp & $90 \%$ \\
\hline
\end{tabular}




\subsection{Kết quả và trao đổi}

Sau khi đã đưa ra quy trình đánh giá, các tiêu chí và phương pháp đánh giá cụ thể cho từng học phần của năm học tương ứng mỗi giảng viên phụ trách. Giảng viên được phân công việc điều phối tổng hợp kết quả đạt được của các SO theo 2 phương pháp (trực tiếp, gián tiếp) như bảng 4.

Bảng 4: Kết quả đạt được các chuẩn đầu ra theo ABET (Năm học 2019-2020)

\begin{tabular}{|c|l|c|c|c|c|c|c|c|}
\cline { 3 - 9 } \multicolumn{2}{c|}{} & \multicolumn{7}{c|}{ Chuẩn đầu ra (\%) } \\
\hline \multicolumn{2}{|c|}{ Phương pháp, học kì } & SO1 & SO2 & SO3 & SO4 & SO5 & SO6 & SO7 \\
\hline \multirow{3}{*}{ Trực tiếp } & Học kì 1 & 72.4 & 86.5 & 82.4 & 87.5 & 100 & 80.7 & 85.7 \\
\cline { 2 - 9 } & Học kì 2 & 93.5 & 90.3 & 91.4 & 88.3 & 86.1 & 79.6 & 93.0 \\
\hline \multirow{2}{*}{ Gián tiếp } & Học kì 1 & 70.3 & 86.1 & 96.1 & 98.3 & 100.0 & 86.3 & 86.3 \\
\cline { 2 - 9 } & Học kì 2 & 95.0 & 91.3 & 94.8 & 88.7 & 88.3 & 80.6 & 96.0 \\
\hline
\end{tabular}

Khi có được kết quả trên, Ban chủ nhiệm Bộ môn nhóm họp các thầy cô để rà soát lại từng học phần để chi tiết hóa kết quả ứng với mỗi $\mathrm{SO}$ để đề xuất phương án cải tiến. Kết quả báo cáo cũng chuyển sang phòng đào tạo để phối hợp thực hiện trong năm học tiếp theo.

\subsubsection{Phuơng pháp trục tiếp}

Phần lớn sinh viên đều đạt chuẩn đầu ra đối với học kì 1 và 2 cho năm học 2019-2020 khi đối sánh với kì vọng $70 \%$ sinh viên đạt ngưỡng đề ra. Do đó, Ban lãnh đạo bộ môn và khoa quyết định đưa ngưỡng kì vọng lên $75 \%$ cho năm học tới (2020-2021). Ngoài ra, có thể thấy được rằng kết quả đối với $\mathrm{SO}$, $\mathrm{SO}$ của học kì 2 thấp hơn so với học kì 1 , trong khi đó kết quả đối với các $\mathrm{SO}$ khác thì ngược lại. Điều này có nghĩa là quá trình giảng dạy đã nâng cao kì vọng cho phần lớn các $\mathrm{SO}$, nhưng đương nhiên điều này cũng còn tùy thuộc vào mỗi học phần. Thực tế, sau khi rà soát chi tiết kết quả của mỗi học phần trong mỗi $\mathrm{SO}$ thì thấy rằng có một số học phần vẫn không đạt kì vọng mặc dù con số trung bình của cả SO là đạt. Phân tích này cho phép Ban lãnh đạo Bộ môn và khoa làm việc cụ thể với giảng viên để tìm ra điểm tồn tại để có biện pháp khắc phục phù hợp, đảm bảo tính cải tiến cho giai đoạn tiếp theo. Ví dụ đối với học phần kĩ thuật khai thác sử dụng đánh giá SO1, nhưng kết quả đạt được của sinh viên của $59,7 \%$. Sau khi phân tích, giảng viên phụ trách học phần này để xuất giải pháp cải tiến như: Động viên sinh viên chuẩn bị kĩ các kiến thức về toán, cơ sở kĩ thuật, đồng thời trong quá trình dạy sẽ tăng cường bài giảng tập trung vào các ví dụ liên quan trực tiếp tới vấn đề kĩ thuật. Ngoài ra, giảng viên sẽ tăng cường hướng dẫn sinh viên cách tìm, đọc tài liệu.

\subsubsection{Phuơng pháp gián tiếp}

Tương tự kết quả của phương pháp trực tiếp, 7 chuẩn đầu ra của sinh viên đều đạt kì vọng trên $70 \%$. Tuy nhiên, đây cũng là lần đầu tiên bộ môn triển khai đánh giá chuẩn đầu ra trên cơ sở ý kiến chủ quan của người học. Mặc dù các năm trước đó cũng lấy khảo sát nhưng nội dung câu hỏi tập trung chủ yếu vào kĩ năng và nội dung giảng dạy mà chưa cụ thể hóa theo các $\mathrm{SO}$. Đồng thời, cũng cần chú ý rằng các giảng viên cũng đã phổ biến tới sinh viên các chuẩn đầu ra liên quan tới câu hỏi trong phiếu khảo sát, nhưng thực chất kết quả chỉ ra một số 
sinh viên vẫn chưa hiểu hết nội dung của $\mathrm{SO}$, điều này phần nào ảnh hưởng tới chất lượng khảo sát. Do đó, bộ môn có kế hoạch từ bắt đầu năm học tới, sẽ tổ chức gặp mặt tất cả sinh viên để phổ biến và trả lời các câu hỏi của sinh viên liên quan tới chuẩn đầu ra, tương quan với các nội dung trong đề cương chi tiết của học phần. Tương tự như kết quả của phương pháp trực tiếp, khi tiến hành phân tích cho thấy kết quả đạt được SO1 của học phần kĩ thuật khai thác là $65,7 \%$. Điều này có nghĩa là có sự phụ hợp giữa sự đánh giá của giảng viên thông qua đánh giá các hình thức và tự cảm nhận tiếp thu của người học.

\section{Kết luận}

Thông qua nghiên cứu của nhóm tác giả cho thấy việc kiểm định chất lượng cơ sở giáo dục nói chung và kiểm định chương trình đào tạo nói riêng và vô cùng quan trọng cho các cơ sở giáo dục trong giai đoạn hiện nay. Một mặt giúp cơ sở giáo dục khẳng định được vị thế của mình trong giai đoạn khó khăn thu hút người học hiện nay. Mặt khác, ý nghĩa to lớn là giúp cho chương trình đào tạo được liên tục cải tiến, người học được tận hưởng một sản phẩm hoàn hảo để trang bị hành trang tốt cho mình khi bước vào thị trường lao động.

Trong số các kiểm định chương trình đào tạo, nhóm tác giả nhận thấy kiểm định theo bộ tiêu chuẩn $\mathrm{ABET}$ là một lựa chọn tốt cho các ngành kỹ thuật. Cơ sở giáo dục có chương trình đạt kiểm định $\mathrm{ABET}$ sẽ là lợi thế rất lớn trong quá trình tuyển sinh, và cung cấp cho người học một tấm vé giá trị được công nhận toàn cầu.

Áp dụng quy trình đánh giá chuẩn đầu ra theo bộ tiêu chuẩn ABET mới cho chương trình kỹ thuật dầu khí của Trường đại học Dầu khí Việt Nam cho thấy phần lớn sinh viên đều đạt chuẩn đầu ra. Phương pháp đánh giá áp dụng là trực tiếp và gián tiếp, có nghĩa là dựa trên cơ sở đánh giá quá trình học tập, thi cử do giảng viên thực hiện, kết quả tự đánh giá của học khi kết thúc học phần. Quá trình đánh giá có sự tham gia của tất cả các bên liên quan, từ người học - giảng viên - bộ môn - khoa - phòng đào tạo - bộ quản đảm bảo chất lượng - cựu sinh viên - người sử dụng lao động. Kết quả đánh giá người học dựa trên chuẩn đầu ra là thông số đầu vào tốt để giúp người vận hành chương trình nhận ra những bất cập và thực hiện cải tiến phù hợp.

\section{Tài liệu tham khảo}

[1] Đinh Ái Linh. Nghiên cứu kiểm định chất lượng các chuyên ngành kĩ thuật ABET của Hoa ki. Tạp chí Khoa học ĐHQGHN. 2014; 2(30): 44-50.

[2] https://Www.abet.org/accreditation/ [2020-11-1].

[3] Nguyễn Hữu Lộc. Đào tạo theo phương pháp tiếp cận CDIO. NXB. ĐHQG TP. HCM, 2018.

[4] Cao Hoàng Trụ. ABET: Mục tiêu và Động lực của việc Đổi mới các Chương trình Đào tạo về $\mathrm{Ky}$ thuật và Công nghệ. [ww.cse.hcmut.edu.vn/ tru/ABET/towardsABET-CHT- BKHCM.pdf 
[5] D. D. Dill and M. Soo. Academic quality, league tables, and public policy: A cross-national analysis of university ranking systems. Higher Education, Vol. 49(4), pp. 495-533, Jun 2005. [Online]. Available: https://doi.org/10.1007/s10734-004-1746-8. 